\title{
Assessment of Ankylosing Spondylitis Based on the Theory of Yin and Yang in Traditional Chinese Medicine
}

\author{
Yuan Cheng1, Jichao Yin'², Xinglv Hu33, Penggang Xu4, Yingchun Liư ${ }^{4}$, Chengxiang Lei5 \\ ${ }^{1}$ Shaanxi University of Traditional Chinese Medicine, Xianyang 712046, Shaanxi Province, China \\ ${ }^{2}$ Xi'an Health School, Xi'an 710000, Shaanxi Province, China \\ ${ }^{3} \mathrm{Xi}$ 'an Hospital of Traditional Chinese Medicine, Xi'an 710000, Shaanxi Province, China \\ ${ }^{4}$ The Fifth Hospital of Xi'an, Xi'an 710000, Shaanxi Province, China \\ ${ }^{5}$ Baoji High-tech Hospital, Baoji 721013, Shaanxi Province, China \\ *Corresponding author: Jichao Yin, 444604786@qq.com
}

\begin{abstract}
The theory of yin and yang in Chinese medicine signifies everything and can be used to divide everything in the world. The diagnosis and assessment of ankylosing spondylitis in Western medicine can also be divided by yin and yang. The current technologies used in Western medicine for detecting ankylosing spondylitis is summarized, divided, and reasoned with the philosophical views of yin and yang in traditional Chinese medicine.
\end{abstract}

Keywords: Traditional Chinese medicine; Ankylosing spondylitis; Yin and yang theory

Publication date: September 2021; Online publication: September 30, 2021

\section{Yin and Yang theory as a simple and broad ancient Chinese philosophy}

They are descriptions of the fundamental factors behind the laws of nature in ancient Chinese civilization, which promote the development and changes of the laws of nature. The principle of the yin and yang theory is widely used in every field of social life. All things in the universe can be divided into two categories according to their attributes: yin and yang. Yang has the characteristics of being vigorous, upward movement, growing, extroverted, stretched, clear, and active. In traditional Chinese medicine, yang represents the human body. All the functional states of invisible yang include excitement, activity, exercise, warmth, exuberance, etc. Yin has the characteristics of weakness, downward movement, contraction, elimination of accumulation, and quietness as far as traditional Chinese medicine is concerned. Yin represents the state of existence of all tangible yin bodies and substances in the human body, such as inhibition, quietness, lubrication, coldness, nutrition, filling, etc. Traditional Chinese medicine uses yin and yang, a macroscopic abstract concept of unity of opposites, to explain the different physiological functions and forms of the human body, as well as the correlation between them.

Ankylosing spondylitis (AS) is a common yet complex disease in orthopedics. It is a combination of ankylosis and spondylitis. It is a chronic and inflammatory rheumatic disease, in which it is characterized by pain and structural dysfunction. The pathogenesis of this disease has not been clearly understood. Most scholars believe that it is related to genetic factors, infection, immunological factors, endocrinological factors, etc. ${ }^{[1]}$. In recent years, the diagnosis, treatment, and evaluation of ankylosing spondylitis have been rapidly developing in Western medicine, and tremendous progress has been made especially in evaluation. 
Although the curative effect of traditional Chinese medicine in the treatment of ankylosing spondylitis is accurate, it lacks the characteristics of traditional Chinese medicine. This article dialectically uses the theory of yin and yang in traditional Chinese medicine with regard to the evaluation methods for AS used in Western medicine.

\subsection{X-ray - Yin-in-yin}

The diagnosis of AS at home and abroad is mostly based on the New York diagnostic criteria revised in $1984^{[2]}$. The standard requirement is the diagnosis of bilateral or unilateral sacroiliitis from an X-ray film. Based on X-ray films, the degree of the sacroiliac joint disease can be divided into 5 grades. The main observations in X-rays are abnormal tissue erosion, hyperplasia, and sclerosis of the anatomical structures of the sacroiliac joint. This can be appreciated by blurring of the subchondral bone margin, bone erosion, blurring of the joint space, increased in bone density, joint fusion, etc. ${ }^{[3]}$. Yue Sheng ${ }^{[4]}$ compared X-ray, computed tomography (CT), and magnetic resonance imaging (MRI) in the diagnosis of AS and showed that the early diagnosis of AS sacroiliac joint disease with X-rays has important reference significance.

The detection range of X-rays includes the chest, abdomen, and bones. The targeted structures in the diagnosis of AS are the sacrum and iliac bones. This technology is not able to detect muscles, ligaments, and other soft tissues. X-rays can only look at yin, but not yang. Therefore, the yin-yang attribute in X-rays is yin-in-yin.

\subsection{MRI - Yin-in-yin}

In AS patients, $50 \%$ to $80 \%$ of patients have bone edema on MRIs ${ }^{[5]}$. The MRI technology can distinguish acute from chronic lesions in AS patients. Hip MRIs can show early hip joint involvement for AS patients, especially those with symptoms of hip joint involvement but negative X-ray findings. Bi Xiaoxiao ${ }^{[6]}$ and other researchers have shown that in the diagnosis and evaluation of the acute inflammatory phase of AS, spine MRI has a high scanning effect in order to correct bone marrow edema and cartilage abnormalities. Compared with other scanning methods, MRI has obvious advantages in showing fat deposition, bone erosion, and tendon enthesitis. At the same time, MRI is also used as a scoring tool for AS. At present, AS spine MRI-a method, Berlin method, and SPARCC (Spondyloarthritis Research Consortium of Canada) method are mostly used. Studies have found that the SPARCC method has the highest sensitivity and consistency.

The fluids of various parts of the human body are collectively referred to as body fluids in traditional Chinese medicine. If soft tissues and bones are divided into yin and yang, soft tissues belong to yang, but as far as the body is concerned, their attributes are still yin. MRI mainly focuses on the yin part of body fluids and soft tissues. Therefore, the yin-yang attribute of MRI is yin-in-yin.

\subsection{Ultrasound - Yang-in-yin}

Ultrasound can display abnormal blood flow signals in the sacroiliac joint. It includes blood flow signals in the sacroiliac joint area, blood flow classification, blood flow display rate, blood flow spectrum classification and distribution, blood flow resistance index, etc. ${ }^{[7]}$. It provides a basis for the assessment of activities in patients with ankylosing spondylitis. In the diagnosis of AS, abnormal blood flow signals can be observed at the sacroiliac joints. Ultrasound is a form of imaging, and the "blood" that this technology mainly observes is yin in TCM. Therefore, no matter how it is divided, the overall attribute of yin and yang in ultrasound belongs to yin. However, the observation of ultrasounds is a dynamic process. Due to the infinite separability of yin and yang, movement and static are obvious signs that distinguish yin and yang, so the yin and yang attribute of ultrasound is yang-in-yin. 


\section{Laboratory examination - Yin}

Laboratory indicators are mainly based on bloods. Chinese medicine believes that the human body is composed of yin, yang, qi, blood, and body fluid. Among them, blood belongs to yin and is the material basis; qi, which is function and energy, belongs to yang. There is a saying in "Difficulty Sutra" that goes, "Qi governs the heart, but blood governs it." Blood plays an extremely important role in human life. Yin and yang are constantly changing over time, where one goes up and down. As far as AS is concerned, the results of the laboratory indicators in the active phase and the chronic phase are quite different. In the active phase, its value is higher than the normal value. The yin-yang attribute of the index is yang whereas the yin-yang attribute of the index lower than the normal value is yin.

\section{Dynamic surface EMG - Yin-in-yang}

Dynamic surface electromyography (sEMG) is of great significance for the evaluation of the neuromuscular system. It has a strong advantage in the diagnosis of muscle diseases and is sensitive in assessing muscle function. The yin and yang attribute of sEMG is yang. However, the observation is not of the whole-body movement but only a certain muscle. First, in terms of a whole and a part, a whole is yang while a part is yin. Secondly, the observed muscle belongs to the human body and the inside is yin. Therefore, the yin and yang attribute of sEMG is yin-in-yang.

\section{Summary and outlook}

"Suwen - The Theory of Yang and Yin should be Xiangxiang" mentioned, "Examine the yin and yang, use softness and rigidity, treat yin in yang disease, and treat yang in yin disease." As far as diagnostic evaluation is concerned, both the diagnosis and treatment belong to the same process. From this point of view, checking yin for yang disease and checking yang for yin disease also have the same scientific nature. According to traditional Chinese medicine, AS belongs to "gubi," "kidney palsy," and other categories. There are four types of symptoms:

(1) kidney deficiency and marrow deficiency type;

(2) yang deficiency and cold coagulation type;

(3) blood stasis block type;

(4) phlegm and dampness block type.

Yin, type 3, and type 4 are empirical and belong to yang. With these, better diagnostic and evaluation methods can be chosen for different types of symptoms. The symptoms of the two types of syndromes, kidney deficiency and marrow deficiency as well as yang deficiency and cold coagulation, are often insidious in onset associated with dull pain at the waist and spine, unfavorable with activities, aggravated with climate change, and occurring intermittently. For these two types of syndromes, the course of the disease is longer. Surface electromyography as well as other yin and yang attributes are used to assess patients' subjective activity ability. The treatment should be based on Tongli Meridian, nourishing Yang, and warming the kidney. The positive assessment technology provides supportive assistance for the subsequent treatment, recovery, and functionality of the patient. The main symptoms of the two syndromes, blood stasis block type and phlegm dampness block type, are tingling of joints, pain, which is heavy and fixed in nature, swelling, joint deformity, etc. Therefore, the examination and evaluation should use the technique with the yin-yang attribute being yin. The treatment is to promote blood circulation, remove blood stasis, dredge collaterals, and relieve pain. The purpose of it is to look for the cause, relieve pain, and restore life. As the saying goes, acute treatment is to relieve symptoms, while slow treatment is to cure the 
root causes.

In order for TCM to develop, it needs to integrate modern medical knowledge and make up for its shortcomings. In order to do so, it is necessary to selectively integrate modern medical knowledge, philosophical thoughts, as well as scientific and technological achievements into TCM theories. It is important to realize the compatibility of TCM theories with Western doctors' understanding of pathology and diagnostic measures while using TCM theories, TCM ideologies, and TCM terminologies to express them in order to develop and enrich the field of TCM.

\section{Funding}

This research was supported by the Multi-Center Collaborative Research on the Optimization and Promotion of Integrated Traditional Chinese and Western Medicine Diagnosis and Treatment Plan for Active Ankylosing Spondylitis, Project Number 2020-ZXY-006 Shaanxi Provincial Department of Health, 2020 Shaanxi Provincial Integrated Traditional Chinese and Western Medicine Clinical Collaborative Innovation Project.

\section{Disclosure statement}

The authors declare that there is no conflict of interest.

\section{References}

[1] Ruan X, Lin H, Du P, et al., 2019, Research Progress in Traditional Chinese and Western Medicine for Ankylosing Spondylitis. South China National Defense Medical Journal, 33(03): 221-223.

[2] Martin B, Amy L, 2018, Managing Morbidity and Treatment-Related Toxicity in Patients with Ankylosing Spondylitis. Rheumatology (Oxford, England), (3).

[3] Chinese Medical Association Rheumatology Branch, 2010, Guidelines for the Diagnosis and Treatment of Ankylosing Spondylitis. Chinese Journal of Rheumatology, 14(8): 557-559.

[4] Yue S, 2020, The Application Value of X-Ray CT and Magnetic Resonance Imaging in the Diagnosis of Early Ankylosing Spondylitis Sacroiliac Joint Disease. Journal of Practical Medical Techniques, 27(01): 38-40.

[5] Isabelle CV, Maria-Antonietta DA, Damien L, 2011, Role for Imaging Studies in Ankylosing Spondylitis. Joint Bone Spine, 78(2).

[6] Bi X, 2020, The Diagnostic Value of Whole Spine MRI for Acute Inflammation of Ankylosing Spondylitis. Psychological Monthly, 15(06): 197.

[7] Lin F, Wang Y, Yuan S, et al., 2019, Preliminary Application of Ultrasound in the Evaluation of Sacroiliac Joints in Patients with Active Ankylosing Spondylitis. Modern Medical Imaging, 28(08): 1715-1718. 